\title{
Medida asociativa y de reforma a la ley de contratacion para ingenieros civiles y arquitectos que permita formar pequeña y mediana empresa para contratar con el estado y poder obtener experiencia laboral
}

\author{
Associative method and reform of the law of recruitment \\ for, civil engineers and architects form small and medium \\ company to hire with the state and to get experience labor
}

DOI: 10.17981/mod.arq.cuc.23.1.2019.02

Artículo. Fecha de Recepción: 04/20/2019. Fecha de Aceptación: 07/21/2019.

Millar A. Martinez G.

Asociacion de municipios de la subregion de la Cienaga Grande Santa Marta. Santa Marta (Colombia). millaradolfomartinez@hotmail.com

Para citar este artículo:

Martínez, M. (2019). Medida asociativa y de reforma a la ley de contratacion para ingenieros civiles y arquitectos que permita formar pequeña y mediana empresa para contratar con el estado y poder obtener experiencia laboral, MODULO ARQUITECTURA CUC, vol. 23, no. 1, pp. 23-38, 2019. DOI: http://doi.org/10.17981/mod.arq.cuc.23.1.2019.02

\section{Resumen}

El presente Artículo investigativo está enfocado en desarrollar y crear las medidas y condiciones normativas flexibles para profesionales egresados de las carreras de Ingeniería Civil y Arquitectura, con el fin de que puedan participar en los procesos de selección y contratación en obras civiles públicas del Estado, y establecer la posibilidad de crear una Pequeña y Mediana empresa de Ingenieros Civiles y/o Arquitectos para contratar obras de mínima y menor cuantía, de esta manera poder incursionar en los proyectos de construcción y desarrollar en lo personal lo aprendido; ya que debido a las constantes modificaciones en materia de infraestructura y el desarrollo tecnológico en Colombia, el nivel de exigencia es mayor por parte del Estado hacia los contratistas. Utilizando como herramienta investigativa la entrevista con un interregonte puntual que proporcione respuestas claras de lo que se quiere investigar, se tiene como resultado que los factores que impiden a los recien egresados de esas facultades incursionar en la Contratacion Estatal son la falta de experiencia y los requisitos poco felxibles de los pliegos de Condiciones, logrando como paso importante en materia de normatividad en contratacion la creacion de los pliegos tipo. Teniendo como conclusion que la Ingenieria Civil y la Arquitectura se posicionan entre las carreras de mayor demanda en el país, pero con el agravante que la creacion de pliegos tipo resulta insuficiente porque aun los requisitos exigidos son muy altos para los recien egresados.

Palabras clave: Ingenieros Civiles; Arquitectos; Contratación; Estado; Pliegos tipo

\section{Abstract}

The present investigative article is focused on developing and creating the flexible normative measures and conditions for graduates of civil engineering and architecture careers, in order to participate in the selection and contracting processes in public civil works of the State, and to establish the possibility of creating a Small and Medium Company of Civil Engineers and / or Architects to contract works of minimum and minor quantity, in this way to be able to venture into the construction projects and develop in the personal what they have learned; since due to constant changes in infrastructure and technological development in Colombia, the level of demand is greater by the State towards contractors. Using as an investigative tool the interview with a punctual interregonte that provides clear answers of what is wanted to investigate, it has as a result that the factors that prevent the recent graduates of these faculties from entering the State Contracting are the lack of experience and the requirements little felxibles of the specifications of conditions, achieving as an important step in terms of regulations in hiring the creation of type sheets. Having as conclusion that the Civil Engineering and Architecture are positioned among the careers of greater demand in the country, but with the aggravating circumstance that the creation of type sheets is insufficient because even the requirements are too high for recent graduates.

Keywords: Civil engineers; Architect; recruitment; State; Sheets type

(C) The author; licensee Universidad de la Costa - CUC. 


\section{INTRODUCCIÓN}

Los profundos cambios ocurridos en Colombia en materia de infraestructura a nivel vial, portuario, obras públicas, viviendas de interés, y debido a la modernización del Estado y a la incorporación de la tecnología de punta en las obras de construcción; la inversión de capital privado, tanto nacional como extranjero, ha hecho más exigente el conocimiento y la experiencia de quienes participan en los procesos de contratación por la calidad de las obras y la inversion que se expone en cada uno de estos proyectos. Esto ha revolucionado igualmente el sistema de contratación por parte del Estado y los contratistas.

Todas estas modificaciones y exigencias han obligado al Estado y a los contratistas a revisar los sistemas de contratación y enganche de los profesionales Colombianos recién egresados de las Universidades en las facultades de Ingeniería Civil y Arquitectura, en la cual se les exigen los conocimientos consolidados y una experiencia mínima de tres (3) años que les impide la incursión laboral y el acceso a los procesos de contratación; ya que el estado exige garantías en la construcción y calidad de las obras, y el inversionista, la protección y garantía en su inversión; por lo cual se exige la experiencia laboral, además de la edad relativa no mayor de los 30 años para estos profesionales estrechándoles las posibilidades de participación y vinculación en el mercado laboral de la construcción y poder adquirir la experiencia necesaria, la cual se convierte en una dicotomía o ambigüedad entre la experiencia, la edad y el no tener la oportunidad de trabajo.

El desarrollo económico y tecnológico al igual que las crisis financieras de algunos sectores ha creado dificultad en el mercado laboral para muchas empresas afectando de paso a la mano de obra de profesionales recién egresados de las universidades del país.

Un sector de estos profesionales afectados, que a pesar del dinamismo de la construcción en la economía del país es el de los recién egresados de las facultades de Ingeniería Civil y Arquitectura, a los cuales en muchos casos no cuentan con las posibilidades de entrar en el mercado laboral nacional, por muchos factores los cuales vamos a ir analizando en el presente trabajo de investigación.

Fenómenos que vienen incorporados a las políticas laborales de las empresas y a los cuales se suman las normas legales de contratación establecidas por el Estado, como es la experiencia laboral y la edad mínima de enganche laboral que deben tener los jóvenes para tener la posibilidad de incorporarse al sistema laboral y productivo de nuestra economía. Uno de ellos es el Estatuto General de Contratación de la Administracion Pública o Ley 80 (1993), que establece una serie de requisitos para poder contratar con el Estado y a la cual se le han hecho reformas, pero las mismas no han sido en beneficio del trabajador o pequeña empresa, mucho menos de los profesionales recién egresados; los cuales por el ritualismo de la contratación pública y las obligaciones y responsabilidades 
añadidas solo con el ánimo de evitar la corrupción, los profesionales de la Ingeniería Civil y la Arquitectura quedan al margen de participar en la contratación pública con el Estado, por no cumplir con los requisitos que exige la ley en esta materia y estar metida la misma en una maraña de interpretaciones jurídicas que complica dicha participación. Sobre el particular plantea Gómez Lee, Ex - Secretario General de la Procuraduría y citado por Salomón (2008) en un artículo sobre reforma a la contratación pública en la revista Derecho y Realidad:

En Colombia las reglas contractuales, desde el decreto Ley 150 de 1976, son un juego de fichas gigantes que, en vez de contribuir a la estabilidad jurídica y a la democratización del derecho, conforman una maraña de normas que solo saben aplicar los especialistas. Las piezas de este rompecabezas en contratación son más de 1500 reglas originadas en las siguientes fuentes materiales: las 2 leyes, 80 de 1993 y 1150 de 2007 con 114 articulos. Todos los reglamentarios que el 066 de 2008 dejo vigentes, para un total de 30 decretos con cerca de 299 artículos (Salomón, 2008, p. 175).

Con relación a lo expresado por el Dr. Gómez Lee, podemos observar que no solo vuelve denso la interpretación, sino que complica y les quita oportunidades a profesionales sin experiencia y con escaso capital como los recién egresados, para darles seguridad y confianza a los empresarios y al Estado a la hora de contratar y exponer sus recursos; por lo cual concluye con su análisis al respecto:
Si las actividades de creación de normas, no es técnica y sistemática, ni ofrece oportunidades de participación ciudadana, el estado carece de seguridad jurídica, al derecho se le resta certeza, efectividad y validez. Igual que la dispersión y proliferación de normas son un alto riesgo, que facilita la corrupción e incrementa las fallas del estado y las condenas en su contra" (Salomón, 2008, p. 176).

En los últimos años hemos sido testigos de la vigorosa revolución de las obras de infraestructura en el país; lo cual exige mayor número de profesionales y mano de obra que aporte su conocimiento al desarrollo y productividad del mismo. Este dinamismo de la economía y la llegada de inversionistas extranjeros al país vuelve más competitiva la mano de obra del profesional Colombiano y enfrentándose a una nueva problemática como lo es el fenómeno del desplazamiento forsozo al que se ha visto perjudicado el profesional Venezolano con capital, buena preparación y experiencia en muchos de ellos; lo que incrementaría la competitividad para los egresados de Ingeniería Civil y Arquitectura en nuestra región y la ciudad.

El modelo económico del país requiere cambios, ya que el mismo ha empobrecido el trabajo de los Colombianos, y la política laboral de los gobiernos sigue siendo precaria en materia de empleo, muy a pesar de las diferentes reformas laborales y tributarias que se han presentado en los últimos años; los resultados no han sido los esperados por la introducción de más impuestos, que, en vez de abaratar la mano de obra para el empresario, los mismos atenta contra la estabilidad laboral. 
Los avances tecnológicos, el desarrollo económico, la sociedad del conocimiento y la globalización en vez de generar mano de obra y participación de los profesionales recién egresados de las facultades de Ingeniería Civil y Arquitectura de la ciudad y la región y en la llamada revolución de la infraestructura vial y pública del país; estos ven disminuida su participación laboral y su vínculo de contratación con el Estado, por dos fenómenos que se han ido radicalizando en el mercado laboral colombiano y que le afecta particularmente a estos dos sectores universitarios. La experiencia laboral en el sector, la edad mínima que tienen los jóvenes para vincularse a una empresa colombiana laboralmente, y a ella le añadimos la excesiva reglamentación del Estatuto General de Contratación de la Administración Pública o Ley 80 (1993) y sus decretos reglamentarios, las excesivas cargas Tributarias, al igual que las cuantías financieras exigidas para grandes obras o mega proyectos; en este sentido la vieja preocupación por la incursión laboral de este grupo de profesionales sigue latente y su aporte de conocimiento al desarrollo y productividad del país por falta de oportunidad se limita; y es cada día más preocupante en la medida que su edad cronológica avanza, su economía privada no la desarrolla; por lo cual pasa a engrosar otro oficio para el cual no se preparó, creando así mayores niveles de informalidad laboral. Ante estos hechos evidentes surge la posibilidad de hacer una propuesta asociativa por parte de los profesionales antes mencionados de agruparse a través de una pequeña y mediana empresa (Pymes) que les permita el acceso a la contratación pública, licitar, contratar y ejecutar obras de minima y menor cuantía; teniendo en cuenta que las (Pymes) se han convertido en el motor del desarrollo económico de muchos países del mundo; por lo cual expresa Ramírez (2005):

Los modelos educativos y empresariales deben ser repensados, demandan nuevas y creativas propuestas que satisfagan las reales de nuestros empresarios Pymes y emprendedores, pero para ello se requiere hacer un diagnóstico de los factores y elementos materiales y legales que permitan flexibilizar el Estatuto de Contratación, crear una normatividad orientada a que estos profesionales tengan la posibilidad de enganche en pequeñas obras de construcción y a ganar la experiencia necesaria en su campo de estudio, teniendo igualmente la posibilidad de acceso a la contratación pública y laboral con el Estado (p. 5).

Con el presente trabajo de investigación se intenta contribuir para establecer la posibilidad de crear una Pequeña y Mediana empresa de Ingenieros Civiles y/o Arquitectos para contratar obras de mínima y menor cuantía con el estado, y desde el plano académico buscar la viabilidad legal y financiera de la posibilidad de reformar el Estatuto de Contratación Pública Colombiano, orientado a mejorar la situación laboral de los profesionales recién egresados de los claustros universitarios de las facultades de Ingeniería Civil y Arquitectura, los cuales muchas veces no pueden desarrollar sus conocimientos y prácticas por la imposibilidad de enganche en el mercado laboral; al exigírsele experiencia y edad mínima 
requerida, asi como otros requisitos legales difíciles de cumplir por estos al inicio de sus carreras. Además, propender porque los grandes problemas sociales y laborales para el desarrollo del país deben exponerse y discutirse desde la academia, y quien más que aquellos profesionales que se sientan afectados con la problemática laboral del empleo, teniendo las competencias y el conocimiento para participar en los destinos y grandes decisiones de la nación.

\section{Marco Teorico}

Legislacion en materia de Contratacion Estatal:En Colombia el proceso de licitación pública para realizar la selección de contratistas en obras públicas se rige por la Ley 80 (1993); esto, sin olvidar que uno de los aspectos fundamentales de la modificacion a esta ley fueron los modelos de selección objetiva, donde ya no se habla de la licitacion y la contratacion directa sino que tambien aparecen en el escenario el concurso de méritos y la selección abreviada, siendo lo anterior modificado por la Ley 1150 (2007) la cual expidió el Estatuto de la Administración de la Contratación pública en el país.

La Ley 1882 (2018, art. 1, parr. 3) establece:

En los procesos de licitación pública para seleccionar contratistas de obra pública, las entidades estatales deberán publicar el informe de evaluación relacionado con los documentos de los requisitos habilitantes y los requisitos que sean objeto de puntuación diferente a la oferta económica incluida en el primer sobre, dentro del plazo establecido en el pliego de condiciones (Ley 1882)
Es aquí donde se presenta la problemática sobre el proceso de selección en el cual se busca a profesionales con cierto grado de experiencia lo cual ocasiona que Ingenieros Civiles y Arquitectos quienes culminan sus carreras se les dificulta la incursión en el mundo laboral.

La Constitución Colombiana (1991, art. 26), plantea la capacidad que tiene el Estado de vigilar, inspeccionar el ejercicio de los profesionales del país. Matallana (2005) en su Manual de Contratación Pública, plantea:

Dentro de los procesos de selección de contratación donde se exija experiencia profesional se hace necesario revisar los ordenamientos que regulan el ejercicio de la profesión; tal es el caso de la arquitectura y las ingenierías, máxime con la apertura de la contratación en el ámbito internacional, en donde algunos eventos se nombran directores o se contrata apoyo técnico de personal profesional extranjero (p. 62).

Aquí podemos observar un antecedente de solicitud de modificación o regulación para la selección de estos profesionales y su situación con la experiencia. El mismo autor citado señala que el Congreso expidió la Ley 842 de 2003, donde se regula la profesión de la Ingeniería, estableciendo los requisitos que se exige cuando se trate de la contratación de obras públicas o cualquier otro contrato que implique el ejercicio de la Ingeniería, de la acreditación conforme lo previene la ley.

Selección Abreviada de Menor Cuantia 
La modalidad de selección abreviada es un procedimiento sencillo y rápido para escoger al contratista en la adquisición de los bienes, obras y servicios cuyo valor no exceda el diez por ciento (10\%) de la menor cuantía de las Entidades Estatales. Pero tambien, se introduce la mínima cuantía como causal de selección abreviada.

\section{El Concurso de Méritos}

Esta modalidad está determinada para los contratos que tengan como objeto una consultoría, la cual es entendida por la doctrina como:

"Los contratos de consultoría están referidos a los estudios necesarios para la ejecución de proyectos de inversión; diagnósticos; asesorías técnicas de coordinación, control y supervisión, interventoría, asesorías, gerencia de obra o de proyectos; dirección, programación y ejecución de diseños, planos, anteproyectos y proyectos (eventos todos en los que, se reitera, el interventor deberá impartir sus órdenes o sugerencias por escrito, enmarcándolas dentro de los términos del respectivo contrato); lo mismo que los concursos para la selección de consultores de diseños, planos, anteproyectos y proyectos arquitectónicos (Salomon, 2008, p. 175).

Teniendo en cuenta lo anterior, la propuesta consiste en crear una normatividad o modificar la existente con el objeto de que los egresados de la Ingeniería y Arquitectura, puedan crear o asociarse en pequeñas empresas para poder participar en los procesos de selección de contratistas con el estado en aquellas obras de menor y minima cuantía y en aquellos proyectos de consultoria que se rigen bajo el modelo de Concurso de meritos. Estableciendo una regulación por parte del Estado para garantizar la idoneidad profesional de los egresados de Ingeniería Civil y Arquitectura que les permita su ingreso al mercado laboral y poder adquirir la experiencia exigida por las políticas de las empresas, así como también las plasmadas a través de la Ley 80 (1993) o Manual de Contratación en Administración Pública.

La razón fundamental por la cual se presenta esta propuesta que va dirigida a los Ingenieros Civiles y Arquitectos recién egresados, es la falta de oportunidades que se les presenta en un mercado laboral sumamente competitivo y exigente en materia legal; este instrumento debe servir de herramienta organizacional que les permita tener fuerza legal para poder canalizar a través de pequeñas asociaciones aquellos contratos de menor cuantía que ofrezca el Estado colombiano y les favorezca y fortalezca para futuros procesos de licitación pública.

El espíritu de asociación tiene como objetivo la participación de todos los agremiados o vinculados en las decisiones públicas que se presenten, en este caso en el mercado de contratación nacional a través de la elaboración de propuestas y proyectos dirigidos al bienestar de los colombianos y para contribuir con el desarrollo de las comunidades en aquellas obras necesarias para el progreso y avance del país.

Participar en igualdad de condiciones en los pliegos de contratación a través de una normatividad flexible y competitiva con aquellas grandes firmas y asociaciones que se llevan los grandes 
contratos, y buscar políticas diferentes de financiación con el apoyo del gobierno y porque no del sector privado no vinculados con los grandes proyectos nacionales. Como las llamadas alianzas Público-Privadas APP, que contribuyan con estos profesionales en su desarrollo empresarial y de emprendimiento.

El modelo actual de contratación del Estado, excluye a los recién egresados o nuevos profesionales, y no cumple con el objetivo de ampliar la cobertura de empleo para los egresados, los cuales se quedan por fuera del mercado laboral y optan por recurrir a otro oficio para el cual no se prepararon y no poder tener acceso a la seguridad social para ellos y su familia.

Se aspira que con la presente propuesta se pueda establecer una Reforma a la presente normatividad para que se introduzcan las modificaciones requeridas para darle flexibilidad y participación a estos profesionales y donde exista un espíritu de equidad en la contratación, excluir algunas obligatoriedades que existen en la actual ley, como requisito para poder participar en las licitaciones y contratación tales como la experiencia laboral y la práctica profesional; el capital o patrimonio autónomo, los cuales endurecen la participación de estos noveles profesionales; se abra la posibilidad a la universalidad en la contratación de menor cuantía y pasar de la exclusión a la participación con el estado.

La propuesta entonces plantea la posibilidad de la modificación del Estatuto de Contratación y las leyes existentes, para que a futuro mejore y favorezca la selección de las propuestas de los egresados de Ingeniería Civil y Arquitectura a través de estas pequeñas asociaciones, garantice el empleo y evite a su vez la explotación laboral y profesional por parte de las grandes compañías y el deterioro en sus condiciones laborales.

\section{Metodología}

Teniendo como referencia la preocupación por parte de los recién egresados de las Facultades de Ingeniería Civil y Arquitectura, el compromiso que tienen con su familia y sociedad en pro del mejoramiento económico y la dignidad humana, la evolucion de la contruccion en el pais y el aumento de la competecia profesional nacional e internacional; resulta pertinente investigar ¿Que factores determinan la poca oportunidad que tienen estos profesionales para no contratar con el estado? Por medio del cual se procede a tomar como metodo de investigacion y recoleccion de informacion, la realizacion de entrevistas, conocida como aquella conversacion cara a cara que se da entre el investigador y el sujeto de estudio o entrevistado, a fin de obtener la informacion relevante sobre nuestro tema de estudio, a traves de respuestas verbales dadas por el sujeto de estudio. Para esta entrevista se procede a la elaboracion de un cuestionario con un solo interrogante muy puntal relacionado con el problema propuesto. La entrevista se caracteriza porque como investigador se puede explicar de forma personal el tema que será tratado durante la entrevista. De esta manera, si existen inquietudes por parte del sujeto de estudio, las podrá plantear de forma abierta y le serán resueltas inmediatamente, todo esto asegura que hayan unas 
mejores respuestas. Dentro de la herramienta investigativa de la entrevista se establecio un solo interrogante como se mensiono con anterioridad, el cual tiene como alternativa la selecccion de varios factores que afectan la incursion del recien ergresado a la contratacion estatal, estos factores son los siguientes:

- Falta de Experiencia Especifica

- Edad relativa no mayor a 30 años / Experiencia General

- Requisitos poco flexibles de los pliegos de condiciones

- Excesiva reglamentación del Estatuto General de Contratación y sus decretos reglamentarios

\section{- Otros}

En el plano personal adquiere relevancia desde mi formacion como ingeniero el contribuir, con los colegas y profesionales de la Ingenieria Civil y Arquitectura que no han tenido la posibilidad de acceso al sistema laboral, además de hacer una reflexión sobre la posibilidad de construir un nuevo sistema de contratacion estatal en el cual se le dé la oportunidad a las nuevas generaciones de profesionales de ganar experiencia y participar en el desarrollo del país y tomar decisiones en proyectos innovadores, teniendo en cuenta que tienen los nuevos conocimientos y las últimas tendencia en materia de investigación y desarrollo en construcción de obras públicas, al igual que incentivar y estimular a otros colegas a participar e investigar en una propuesta más sistematizada y coherente con las necesidades de empleo y mano de obra calificada para estos profesionales.
Sin duda que los aportes que arroje este trabajo en el ámbito académico y laboral dinamizaran las posibilidades de incorporación de otras ramas del saber en poder contribuir a la vez con el gobierno para que este pueda mover su poder legislativo en modificar los factores legales que impiden una incorporación real al sector económico y laboral de los recién egresados de Ingeniería Civil y Arquitectura.

Esta propuesta pretende aventurarse en una reflexión sobre una posible modificación de la normatividad y se pueda crear un nuevo desarrolo legal que tenga en cuenta los elementos aquí anunciados para modificar además las reglas de juego de la contratación estatal, que les permita la participación a los profesionales antes mencionados en la investigación.

\section{Delimitación de la Investigación $y$ analisis de muestra}

El empleo, como el trabajo se constituye en una de las mayores necesidades del ser humano, ya que a través de ellos se pueden adquirir los recursos que le posibiliten la subsistencia y el poder proyectarse como un ser humano con dignidad; por lo cual nuestro objeto de trabajo en la presente investigación se limita al análisis de los factores que impiden la no vinculación de los profesionales recién egresados de las facultades de Ingeniería Civil y Arquitectura en Universidades de la ciudad de Barranquilla, pero limitándolo aún más al espacio laboral y empresarial en el cual me desempeño como lo es la Contratación Estatal, es por eso que se plantea como herramienta de 
investigacion la realizacion de entrevistas a una muestra aleatoria de egresados de Ingenieria Civil y de Arquitectura de la Universidad de la Costa y la Universidad del Norte. Si deseamos estimar una proporción, debemos saber (Fisterra, 2019):

1. Nivel de confianza o seguridad (1-a). El nivel de confianza prefijado da lugar a un coeficiente (Za). Para una seguridad del 95\% = 1.96.

2. La precisión que deseamos para nuestro estudio.

3. Una idea del valor aproximado del parámetro que queremos medir (en este caso una proporción). Esta idea se puede obtener revisando la literatura, por estudios pilotos previos. En caso de no tener dicha información utilizaremos el valor $\mathrm{p}=0.5(50 \%)$.

¿Cuántos egresados deberiamos entrevistar o estudiar para conocer los factores que mas limitan la vinculacion de estos a la contratacion estatal?

\section{Nivel de Confianza $=95 \%$;}

Margen de error $=3 \%$

Proporción esperada = asumamos que puede ser próxima al 5\%; si no tuviésemos ninguna idea de dicha proporción utilizaríamos el valor $\mathrm{p}=0,5(50 \%)$ que maximiza el tamaño muestral:

$$
n=\frac{Z_{\mathrm{a}}^{2} * p * q}{d^{2}}
$$

Donde:

$$
\begin{array}{ll}
Z_{a}^{2}= & \begin{array}{l}
\text { 1.96, teniendo en cuenta que la } \\
\text { seguridad es del } 95 \% .
\end{array} \\
p= & \begin{array}{l}
\text { proporción esperada } \\
(\text { en este caso } 5 \%=0.05)
\end{array} \\
q= & \begin{array}{l}
1-\text { p (en este caso } 1-0.05=0.95) \\
\text { margen de error en este caso } \\
\text { deseamos un } 3 \%
\end{array} \\
n= & \frac{1,96^{2} * 0,05 * 0,95}{n \cap 22}=203
\end{array}
$$

\section{Resultados}

De acuerdo con los datos de Mineducación (2019), de los 2960 egresados durante el año 2013, el $91.6 \%$ consiguió trabajo en el 2014, con un salario promedio de $\$ 1.736 .849$ pesos, A su vez, el salario de enganche de un graduado de maestría, supera los $\$ 3,9$ millones (Figura 1). Por otro lado y con datos mas actualizados, las carreraras que toman vuelo en areas de conocimiento demandadas por los estudiantes; la ministra de educación de esa epóca, Cecilia Vélez, resaltó que entre estos años, el 31,3\% de los 1.381.761 títulos (es decir, 425.895) fueron otorgados en programas académicos relacionados con economía, administración y contaduría. El 23,7\% de los títulos (323.303) están relacionados con las áreas de ingeniería, arquitectura, urbanismo y afines; el 18,5\% (252.116) en ciencias sociales y humanas; el 11,1\% (150.902) en ciencias de la educación; el 9\% (123.180) en ciencias de la salud; el 3,3\% (44.706) en bellas artes; el 1,6\% (21.698) en ciencias básicas y el 1,4\% (19.548) en agrono- 
mía y veterinaria. Otro aspecto importante que quiso destacar la jefe de la cartera educativa es que, carreras como ingeniería industrial, Civil, Arquitectura y Medicina son algunas de las que tienen mayor grado de vinculación laboral, es decir, que sus profesionales están siendo muy demandados por el mercado. Igualmente, estas carreras son muy bien remuneradas con respecto a otras que también registran un alto número de graduados. Por ejemplo, de los graduados entre 2001 y 2009 de ingeniería industrial, el 82,5\% está vinculado al sector formal de la economía y tienen un salario promedio de $\$ 1.585 .887$. De los egresados de Medicina, el 90,4\% tiene trabajo y gana en promedio $\$ 2.162 .672$; mientras que de los Ingenieros Civiles y Arquitectos, el 82,9\% está trabajando con un sueldo de $\$ 1.499 .3328$ (Mineducación, 2019).

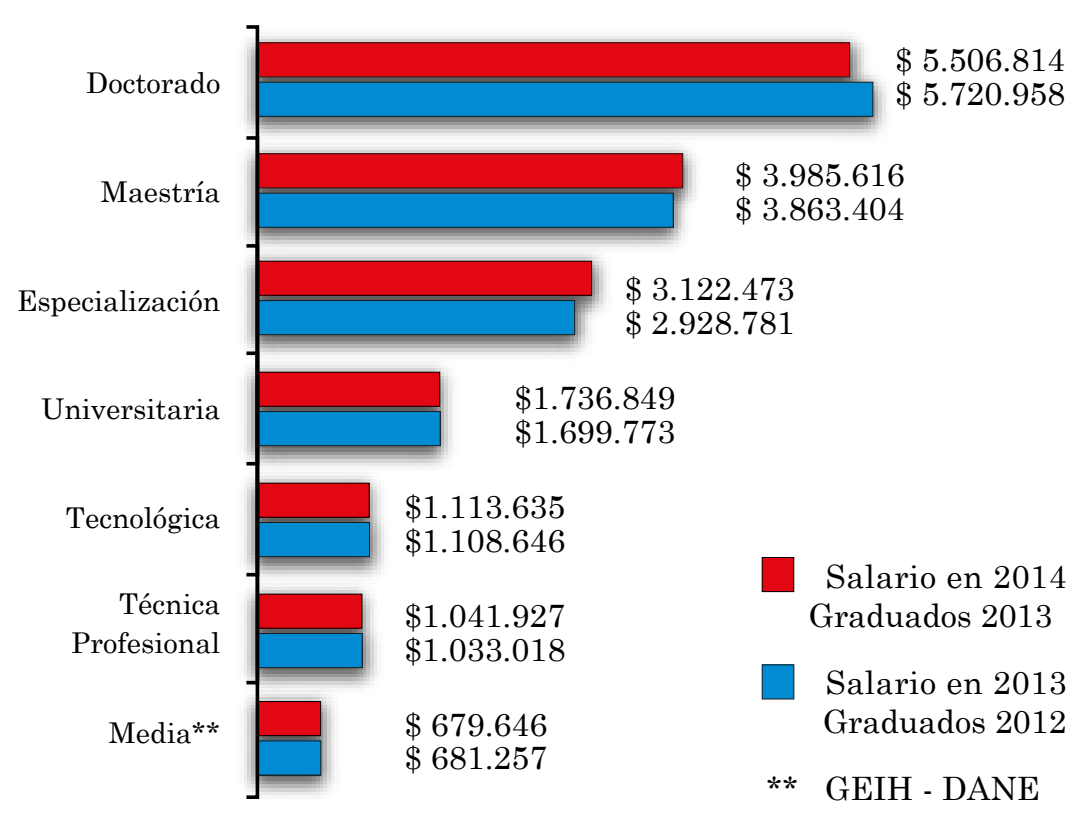

Figura 1. Salario Promedio de los recien graduados (Precios constantes 2014). Fuente: Mineducación, 2019.
Lo anterior posiciona a la Ingeniería Civil y Arquitectura entres las carreras de mayor demanda en el país con una tasa de empleabilidad superior a otras opciones de estudio y que además brinda la posibilidad de salario de enganche acorde con sus conocimientos. De acuerdo con la información obtenida, el mercado laboral en este momento es bueno en Colombia para el sector; debido a que el país va en vía de desarrollo desde hace una década; además de la alta demanda de las infraestructuras que necesitan ser hechas y reparadas. Dicho esto, cabe resaltar que la competencia se hace elevada, pero, la cantidad de oferta no es proporcional a la cantidad de demanda requerida.

Dentro de los resultados arrojados por las entrevista, se puede evidenciar que la informacion recolectada arroja como la Falta de Experiencia y los Requisitos poco Felxibles de los Pliegos de Condiciones, como los factores que imposibilitan la incursion de los profesionales recien egresados a la contratacion estatal, entiendolo como los resultados que mas se repiten dentro de las entrevistas realizadas (Tabla 1 y Tabla 2) (Figura 2 y Figura 3). Los requerimientos que se le hacen a los Ingenieros Civiles y Arquitectos recaen sobre la necesidad de actualizar sus conocimientos, para estar acordes con los avances nacionales e internacionales en materia de construcción como lo es la Norma Sismoresistente, entre otros avances. El Ingeniero y Arquitecto deben tener la habilidad, la capacidad Investigativa, comunicativa en términos técnicos; ofrecer propuestas innovadoras, ser proactivo y poder encargarse de proyectos de alto impacto: sea este público, económico y social. 
TABla 1.

Tabulacion Egresados de ingenieria Civil y Arquitectura Universidad CUC.

\begin{tabular}{|c|c|c|c|}
\hline No. & Pregunta & Respuestas & $\begin{array}{c}\text { Cantidad } \\
\text { Estudiantes }\end{array}$ \\
\hline \multirow{6}{*}{1} & \multirow{6}{*}{$\begin{array}{l}\text { ¿Elija que factores determinan la poca } \\
\text { oportunidad que tienen los profesionales } \\
\text { de Ingenieria Civil y Arquitectura para } \\
\text { no contratar con el Estado? }\end{array}$} & Falta de Experiencia Especifica & 33 \\
\hline & & Edad relativa no mayor a 30 años / Experiencia General & 15 \\
\hline & & Requisitos poco Felxibles de los Pliegos de Condiciones & 29 \\
\hline & & $\begin{array}{l}\text { Excesiva reglamentación del Estatuto General de Contratación } \\
\text { y sus decretos reglamentarios }\end{array}$ & 16 \\
\hline & & Otros & 9 \\
\hline & & Total & 102 \\
\hline
\end{tabular}

Fuente: Autores.

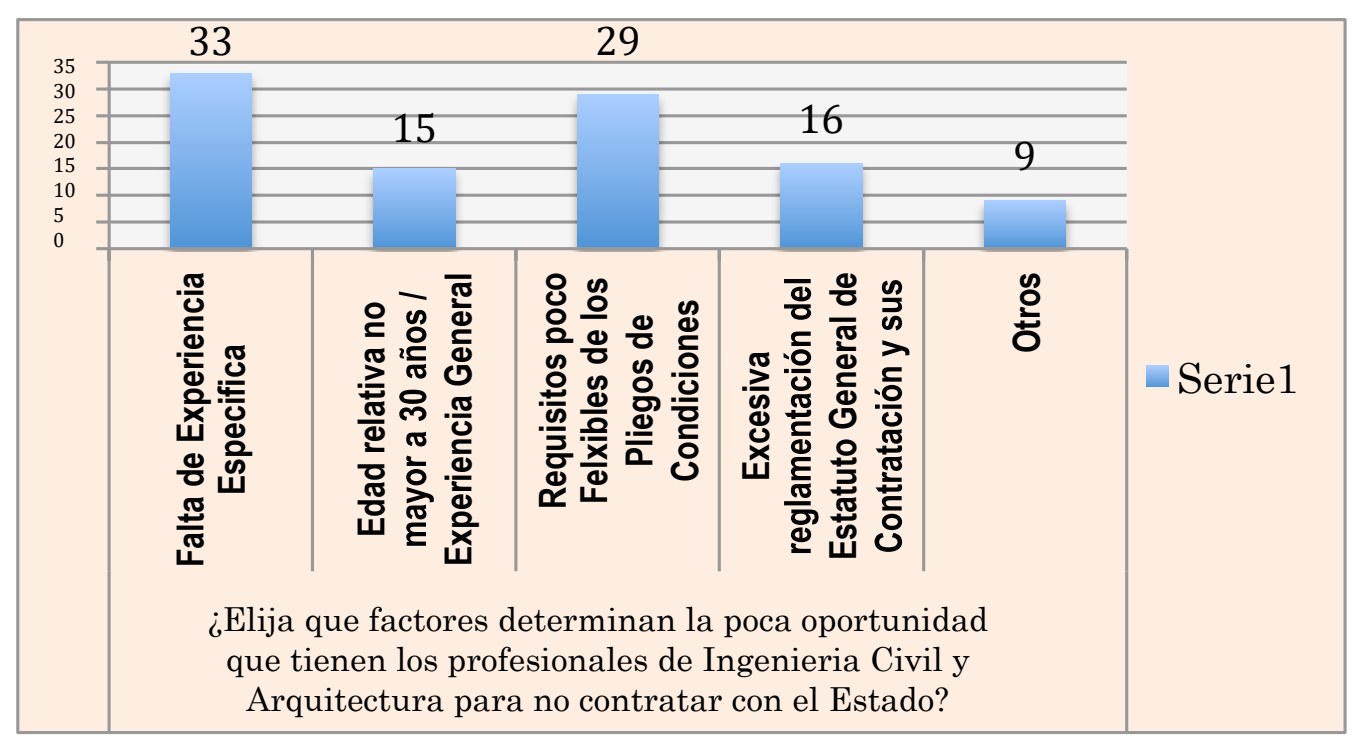

Figura 2. Resultados Factores determinantes. Fuente: Autores. 
TABLA 2.

Tabulacion Egresados Universidad del Norte.

\begin{tabular}{lllc}
\hline No. & Pregunta & Respuestas & $\begin{array}{c}\text { Cantidad } \\
\text { Estudiantes }\end{array}$ \\
\hline & & Falta de Experiencia Especifica & 39 \\
& & Edad relativa no mayor a 30 años / Experiencia General & 8 \\
& ¿Elija que factores determinan la poca & Rortunidad que tienen los profesionales & Requisitos poco Felxibles de los Pliegos de Condiciones \\
1 & $\begin{array}{l}\text { de Ingenieria Civil y Arquitectura para } \\
\text { no contratar con el Estado? }\end{array}$ & Excesiva reglamentación del Estatuto General de Contratación & 36 \\
& & y sus decretos reglamentarios & 11 \\
& Otros & Total & 7 \\
\hline
\end{tabular}

Fuente: Autores.

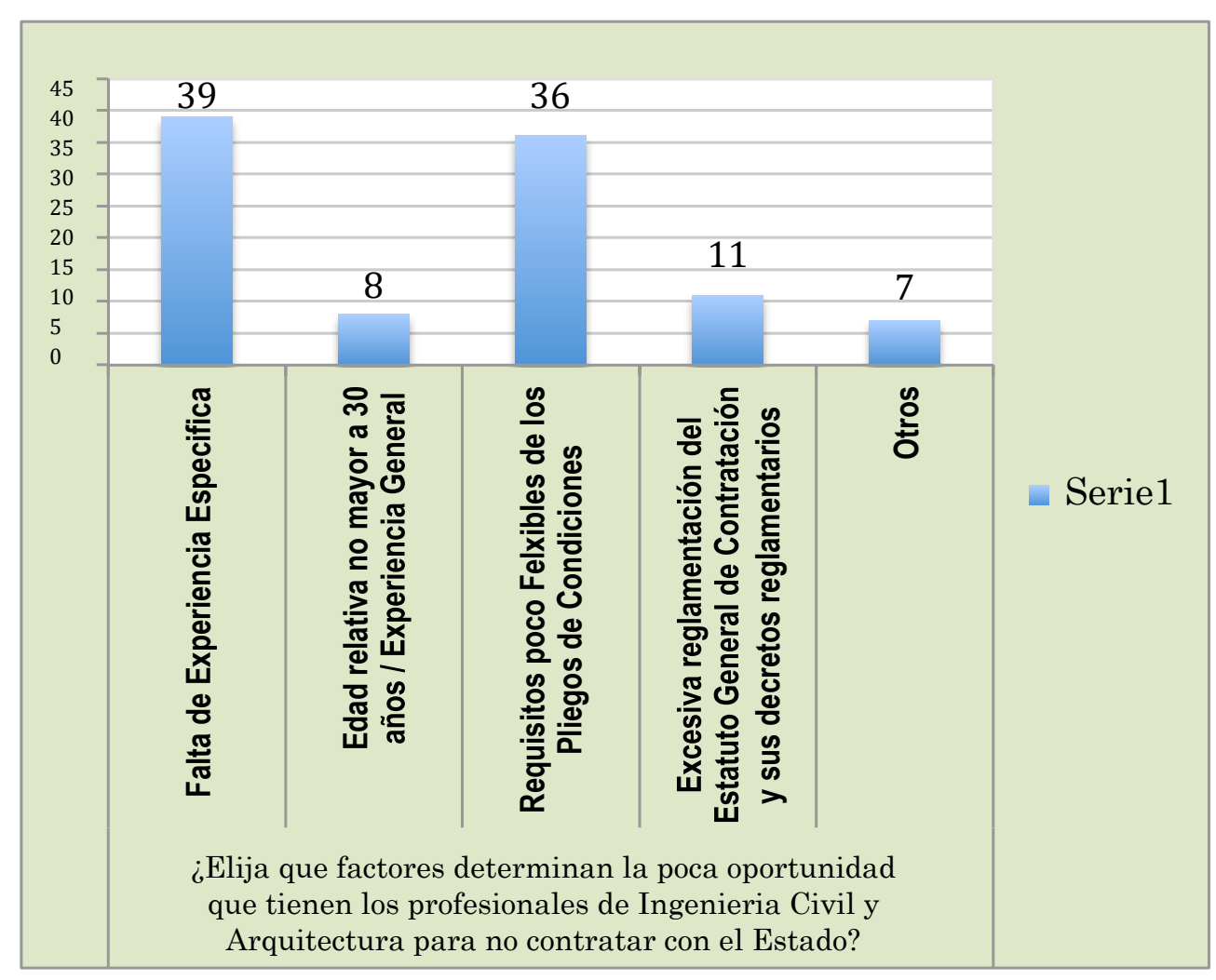

Figura 3. Resultados Factores determinantes. Fuente: Autores. 
En lo respectivo a la normatividad vigente en contratacion y que su propuesta sea modificada, el gobierno aprobo la Ley 1882/2018, la cual plantea la obligación al sector público y a la administración en general a estandarizar los pliegos de contratación para obras públicas en los municipios del país. El pliego tipo evitara la manipulación de la contratación por parte de los grandes oferentes y para que haya pluralidad en la presentación de las ofertas y se favorezca más a las pequeñas y medianas empresas. Esto hace parte de la investigación que estamos proponiendo y abre una puerta para la incorporación de los pliegos tipo en la contratación, se evita la cooptación por parte de los poderosos empresarios y oferentes a la contratación estatal, esto vuelve más transparente la contratación dándo mayor pluralidad a la participación. Ademas de la Ley anteriormente mensionada y creada recientemente, el gobierno nacional inicia la estadarizacion de los pliegos tipo mediante la aprobacion del Decreto 342 (2019), mediante el cual se adiciona una seccion de un capitulo del Decreto 1082 (DNP, 2015), Decreto Unico Reglamentario del Sector Administrativo de Planeacion Nacional (DNP), que establece inicialmente el documento tipo para licitacion de obra pública de infraestructura de transporte, los cuales contienen parametros obligatorios para las entidades estatales sometidas al Estatuto General de Contratación Pública.

En el día a día de un profesional de la Ingeniería surgen ciertos retos que se presentan en cada carrera al momento de ejercer la profesión, en este caso consiste en identificar los factores más relevantes que constituyan los principales retos de la educación de la ingeniería en el contexto de la nueva sociedad del conocimiento. A este primer objetivo se responde con la caracterización del contexto socioeconómico, el contexto laboral, el contexto científico-tecnológico, el nuevo perfil del ingeniero, etc. Se busca entonces plantear estrategias de largo plazo que permitan a los Ingenieros y Arquitectos valorizar una vinculación laboral contractual con el estado y recordar acciones específicas a los sectores profesionales involucrados con la profesión.

\section{Conclusiones}

En Colombia funcionan cientos de programas académicos en 237 centros de educación superior en diversas áreas de Ingeniería que preparan a 125.000 estudiantes ¿cuál es el futuro del Ingeniero y el Arquitecto?. Las universidades necesitan replantear con urgencia los currículos para volverlos más gerenciales que operativos, más humanos que manejador de ecuaciones, y más comunicadores que un ser humano ensimismado en su trabajo. El Ingeniero y el Arquitecto reflejan la importancia de estas profesiónes en la estructura socioeconómica del país y constituyen una de las principales herramientas del crecimiento de las micro sociedades.

La ingeniería y Arquitectura moderna data del siglo XVII, pero fue en las últimas cuatro décadas en que esta actividad adquirió mayor dimensión y profundidad, superando el paradigma de ser unas carreras técnicas, para convertirse en profesiónes 
con grandes alcances humanísticos, sociales, económicos, administrativos y ecologicos.

Con base a los resultados planteados con anterioridad acerca de las estadisticas de enganche laboral de la Ingenieria Civil y Arquitectura que son muy buenas con realcion a otras carreras, cabe resaltar que la investigación plantea la necesidad de buscar la manera de modificar el modelo de contratación del Estado para que pequeños grupos de profesionales asociados en pequeñas empresas o en la creacion de incubadoras puedan participar en la contratación de menor cuantía y minima cuantia; ya que lo que se requiere es que el recien egresado de las Facultades de Ingenieria y Arquitectura no sean conformistas con lograr la incursion laboral en cargos de Supervisores y/o Ingenieros o Arquitectos Residentes, sino que el gobierno les permita participar en la contratacion. Si bien es cierto, el gobierno inicio la transformacion del modelo de contratacion con la creacion de la Ley 1882 (2018) y el Decreto 342 (2019), mediante la implementacion de los pliegos tipo, esto aun resulta insuficiente para que el prefesional egresado de estas facultades pueda lograr participar en algun proceso o modelo de contratacion, ya que aun los parametros exigidos en los pliegos tipo resultan ser muy exigentes y excluyentes para nuestros egresados, lo que imposibilita el poder contratar con el Estado. Teniendo como estrategia la presentación de un modelo de modificación a consideracion del gobierno a través de una iniciativa popular para que el senado tramite la modificación, también se hace necesario que los profesionales involucrados conozcan cuales son las herramientas que necesitan modificación.

\section{RECOMENDACIONES}

Teniendo en cuenta la reinvencion que estan teniendo las incubadoras, ya que fueron las primeras en el apoyo al desarrollo del emprendimiento del pais, se busca el apoyo de las Universidades y las Camaras de Comercio de incentivar a los recien egresados de las Facultades de Ingenieria Civil y Arquitectura para fotrtalecer sus competecncias y buscar el enganche laboral.

Se plantea con este trabajo la necesidad de crear entre los egresados recién graduados de las facultades de Ingeniería y Arquitectura, un modelo asociativo de empresas, para que estos puedan participar en la oferta de obras públicas con el estado; sobre todo en obras de menor y minima cuantía.

Propiciar espacios para la discusión desde la universidad de una iniciativa de modificación actual al modelo de contratación de la nación; que flexibilice los parametros exigidos, para la vinculación de estos profesionales, y hagan carrera en materia de contratación.

Hacer visible esta propuesta a través de actividades de seminarios, foros empresariales y eventos relacionados con la contratación pública y la infraestructura de obra del país y la región; con el objeto final de posesionar una posible marca empresarial desde la asociatividad y cooperación de este grupo de profesionales.

\section{REFERENCIAS}

Fisterra.com (mayo 1, 2019). Determinacion Tamaño Muestral. [Online]. Disponible en https://fisterra.com/mbe/investiga/9muestras/9muestras2. asp 
Matallana, E. (2005). Manual de Contratacion de la Administracion Publica. Reforma de la Ley 80 de 1993. Incluye Decreto 1082 de 2015 (4 ed.). Bogotá, D.C.: Universidad Externado de Colombia.

Ramírez, J. (2005). Pymes mas competitivas. Como pueden afrontar con éxito las pequeñas y medianas empresas el agresivo mundo globalizado de los negocio. (2 ed.). Bogotá, D.C.: Mayol ediciones.

Salomon, J. (2008). La reforma a la contratacion. Derecho y Realidad, (11), 175-176.

República de Colombia. Asamblea Constituyente. (1991). Artículo 26. [Constitución política de Colombia]. Gaceta Constitucional, 1(114). 4. Recuperado de http://babel. banrepcultural.org/cdm/compoundobject/ collection/p17054coll26/id/3850/show/3743/ $\mathrm{rec} / 8$

República de Colombia. Congreso de la República. (16 de julio de 2007). Por medio de la cual se introducen medidas para la eficiencia y la transparencia en la Ley 80 de 1993 y se dictan otras disposiciones generales sobre la contratación con Recursos Públicos. [Ley 1150]. Diario Oficial: 46.691.

República de Colombia. Congreso de la República. (28 de octubre de 1993). Por la cual se expide el Estatuto General de Contratación de la Administración Pública. [Ley 80]. DO: 41.094. Disponible en http://www. secretariasenado.gov.co/senado/basedoc/ ley_0080_1993.html
República de Colombia. MinEducación. (mayo 1, 2019). Situacion laboral de los graduados. Observatorio Laboral para la Educacion. [Online]. Disponible en https://ole.mineducacion.gov.co/portal/

República de Colombia. Presidencia de la República. (11 de enero de 2018). Por la cual se adicionan, modifican y dictan disposiciones orientadas a fortalecer la contratación pública en colombia, la ley de infraestructura y se dictan otras disposiciones. [Ley 1882]. DO: 50.477. Disponible en http://es.presidencia. gov.co/normativa/normativa/LEY 1882 DEL 15 DE ENERO DE 2018.pdf

República de Colombia. Presidencia de la República. (5 de marzo de 2019). Por el cual se adiciona la Seccion 6 de la Subseccion 1 del Capitulo 2 del Titulo 1 de la parte 2 del Libro 2 del Decreto 1082 de 2015, Decreto Unico Reglamentariodel Sector Administrativo de Planeacion Nacional. [Decreto 342]. DO: 50.886. Disponible en http://es.presidencia. gov.co/normativa/normativa/DECRETO $\% 20$ 342\%20DEL\%2005\%20DE\%20MARZO\%20 DE\%202019.pdf

República de Colombia. Departamento Nacional de Planeación. (26 de Mayo de 2015). Por medio del cual se expide el decreto único reglamentario del sector administrativo de planeación nacional. [Decreto 1082]. DO: 49.523. Disponible en https://www.icbf.gov. co/cargues/avance/docs/decreto_1082_2015. htm 


\section{ANEXO A}

Modelo de entrevista

- Medida asociativa y de reforma a la ley de contratacion para ingenieros civiles y arquitectos que permita formar pequeña y mediana empresa para contratar con el estado y poder obtener experiencia laboral

\section{PREGUNTA 1.}

¿Elija que factores determinan la poca oportunidad que tienen los profesionales de Ingenieria Civil y Arquitectura para no contratar con el Estado?

Falta de Experiencia Especifica

Edad relativa no mayor a 30 años / Experiencia General

Requisitos poco Felxibles de los Pliegos de Condiciones

Excesiva reglamentación del Estatuto General

de Contratación y sus decretos reglamentarios

Otros 